Herz 2019 • 44:93-95

https://doi.org/10.1007/s00059-019-4793-8

(c) Springer Medizin Verlag GmbH, ein Teil von Springer Nature 2019

\section{H. Winkels ${ }^{1} \cdot$ S. Baldus ${ }^{2,3}$}

' La Jolla Institute for Immunology, La Jolla, USA

${ }^{2}$ Herzzentrum, Abt. f. Kardiologie, Universität Köln, Köln, Deutschland

${ }^{3}$ Zentrum für Molekulare Medizin Köln CMMC, Universität Köln, Köln, Deutschland

\title{
Impfung zur Behandlung der Arteriosklerose - eine realistische Vision?
}

Atherosklerose, die chronische Entzündung großer und mittelgroßer Arterien, wird in wesentlicher Weise begünstigt durch die erhöhte Bioverfügbarkeit und endotheliale Ablagerung von LDL(„low-density lipoprotein“)-Cholesterin. Folglich haben Statine und gegen Proproteinkonvertase Subtilisin/Kexin Typ 9 (PCSK9) gerichtete Antikörper die Inzidenz akuter kardiovaskulärer Erkrankungen halbiert $[1,2]$. Trotz erfolgreicher Senkung des LDL-Cholesterins besteht weiterhin ein relevantes Risiko für kardiovaskuläre Ereignisse, welches überwiegend inflammatorischen Ursprungs ist. Bereits Rudolf Virchow erkannte die entzündlichen Charakteristika atherosklerotischer Läsionen. Im Jahr 2017 wurde in der Canakinumab Anti-Inflammatory Thrombosis Outcome Study (CANTOS) Infarktpatienten, welche einen erhöhten Serumspiegel des C-reaktiven Proteins aufwiesen, ein inhibierender Interleukin(IL)-1-beta-Antikörper (Canakinumab) verabreicht. Diese therapeutische Intervention reduzierte erfolgreich die Inzidenz sekundärer Myokardischämien [2]. Die Gesamtsterblichkeit war jedoch aufgrund einer herabgesetzten Immunabwehr mit konsekutiven tödlichen Infektionen unverändert. Daher müssen selektivere Therapien zur Bekämpfung des inflammatorischen Restrisikos entwickelt werden.

Impfstrategien erweisen sich hier als prophylaktisches Mittel der Wahl, da Impfungen im Gegensatz zu zytokininhibierenden Antikörpern antigenspezifisch und somit ohne generellen Einfluss auf das körpereigene Immunsystem wirken.

Die Impfung ist ein Meilenstein der medizinischen Errungenschaften, und es werden heute über 27 Infektionskrankheiten und mehrere Krebserkrankungen verhindert. Dies hat signifikant zur gesteigerten durchschnittlichen Lebenserwartung beigetragen. Zweck der Impfung ist es, das Immunsystem spezifisch auf eine Infektion durch Pathogene wie Bakterien oder Viren oder die Ausbildung von Tumorzellen vorzubereiten. Erfolgt dann die tatsächliche Infektion, bildet sich eine schnellere und effizientere „recall response" aus, bei der die Pathogene oder Tumorzellen mit Antikörpern zur Aufnahme durch Phagozyten markiert werden. Weiterhin werden CD4-T-Zellen und CD8-T-Zellen oder Killerzellen aktiviert, sodass Tumorzellen oder virusbefallene Zellen eliminiert werden.

Die erfolgreiche Ausbildung einer adaptiven Immunantwort bedingt die Aktivierung des angeborenen Immunsystems. Im Speziellen überwachen Zellen des angeborenen Immunsystems das periphere Gewebe. Bei Infektion phagozytieren vor allem dendritische Zellen (DC) und Makrophagen das Pathogen, wandern in den lokalen Lymphknoten ein und präsentieren dort Peptide des Pathogens in Haupthistokompatibilitätskomplexen (MHC). Dort reagieren antigenspezifische T-Zellen und initiieren die adaptive Immunantwort. Obwohl es mehrere Kontrollmechanismen zum Schutz des Körpers gegen eine unerwünschte oder überschießende Immunantwort gibt, kann die Präsentation von Autoantigenen zu Autoimmunerkrankungen führen. Tatsächlich wurden DCs und Makrophagen bereits in der nicht atherosklerotischen Aorta gefunden, wo sie über „pattern recognition receptors" wie Toll-like-Rezeptoren aktiviert werden können und nachfolgend in drainierende Lymphknoten einwandern [3].

Mehrere Beobachtungen deuten darauf hin, dass Atherosklerose Autoimmunkomponenten aufweist, welche die Progression der Erkrankung verhindern oder fördern. Mehrere Antigene, unter anderem PCSK9, HSP65 („, heat-shock protein 65") und Apolipoprotein B100 (Apo- $\mathrm{B}_{100}$ ), die Proteinstrukturkomponente des LDL-Cholesterols, wurden in vulnerablen Plaques identifiziert. Atherosklerotische Patienten und Mäuse haben zirkulierende Antikörper, welche gegen oxidiertes LDL (oxLDL) gerichtet sind. Dies deutet auf eine Aktivierung von B-Zellen hin, was die Aktivierung durch CD4-T-Helferzellen impliziert. Oxidierte Phospholipide sind proinflammatorisch und fördern Atherosklerose. Ein natürlich vorkommender Immunglobulin-M(IgM)-Antikörper (E06), gerichtet gegen die Phosphocholinkopfgruppe der oxidierten Phospholipide, verhindert deren Aufnahme durch Makrophagen und verringert Atherosklerose $[4,5]$.

CD4-T-Zellen sind in murinen und humanen atherosklerotischen Plaques aufzufinden. Expressionsanalysen der T-Zell-Rezeptoren (TCR) in atherosklerotischen Läsionen konnten aufzeigen, dass die TCR-Heterogenität herabgesetzt ist, was auf die Akkumulation oligoklo- 
nal expandierter T-Zellen schließen lässt [6]. Interessanterweise wandern CD4-T-Zellen aus atherosklerotischen Mäusen im Gegensatz zu CD4-T-Zellen aus gesunden Mäusen in atherosklerotische Aorten ein und werden dort zur Zytokinproduktion aktiviert [7]. Dies untermauert die Präsenz und Aktivierung atheroskleroserelevanter CD4-TZellen. Weiterhin reagierten T-Zell-Hybridomas aus mit oxLDL-vakzinierten Mäusen vorwiegend auf natives LDL und Apo-B $\mathrm{B}_{100}$ [8]. Dies impliziert, dass bereits in der nicht immunisierten Maus Apo- $B_{100}$-reaktive $\mathrm{T}$-Zellen vorhanden sind.

Kimura et al. konnten kürzlich erstmals demonstrieren, dass gesunde Probanden und nicht atheroklerotische Mäuse CD4-T-Zellen spezifisch für ein Antigen des Apo-B $B_{100}$ (P18) aufweisen [9]. Zur Identifikation wurden fluoreszierende Tetramere entwickelt. Diese Komplexe bestehen aus 4 über Streptavidin miteinander verknüpften MHCII-Monomeren und sind in diesem Fall mit dem P18-Peptid Apo- $B_{100}$ s beladen. P18 war der geeignete Kandidat, da es sequenzhomolog zwischen Mensch und Maus ist und in MHC-Komplexen beider Spezies präsentiert werden kann. Interessanterweise war die relative Anzahl dieser Zellen unverändert zwischen gesunden Probanden und kardiovaskulär erkrankten Patienten. Analyse mittels Durchflusszytometrie ergab jedoch ein verändertes Bild T-Zell-spezifischer Transkriptionsfaktoren. So waren P18reaktive CD4-T-Zellen überwiegend regulatorischer Natur, wohingegen diese Zellen von kardiovaskulär Erkrankten vorwiegend proinflammatorische und somit proatherosklerotische Phänotypen aufwiesen.

Das Vorhandensein von T-Zellen, welche auf atheroskleroserelevante Antigene reagieren, legt die Grundlage zur Entwicklung einer impfungsbasierenden Therapie zur Prävention von Herz-Kreislauf-Erkrankungen. Dabei ist es notwendig, einen regulatorischen T-Zell-Phänotypen $\left(\mathrm{T}_{\mathrm{reg}}\right) \mathrm{zu}$ induzieren und proatherosklerotische T-ZellPhänotypen zu verhindern.

Atherosklerotische Läsionsgrößen wurden erfolgreich in atheroskleroti- schen Mausmodellen nach Immunisierung mit von Apo-B abgeleiteten MHC-II-bindenden Peptiden verringert [10]. Dabei wurde eine protektive Antikörperreaktion gegen das benutzte Antigen detektiert. Diese und andere Berichte haben zu der Entwicklung der GLACIER-Studie geführt. Dabei wurde Patienten mit stabilen atherosklerotischen Plaques, welche cholesterinsenkende Medikamente erhielten, ein gegen oxLDL gerichteter monoklonaler Antikörper intravenös verabreicht. Es wurde jedoch keine Veränderung der atherosklerotischen Plaquegröße beobachtet, was unter Umständen durch das Studiendesign bedingt war [11]. Weiterhin ist es aus Mausmodellen bekannt, dass impfstoffinduzierte Antikörperantworten zur Atheroprotektion allein nicht mehr ausreichen [12]. Andere immunologische und antiatherosklerotische Mechanismen, wie die Induzierung von $\mathrm{T}_{\text {regs }}$ oder die Expression des entzündungshemmenden Zytokins IL-10, sind notwendig. So löste die Immunisierung mit dem Apo-B ${ }_{100}$-Peptid P101 IL-10Produktion in CD4-T-Zellen aus [13]. Die Immunisierung mit Apo- $\mathrm{B}_{100}$-Peptid P18 erhöhte das Vorhandensein P18spezifischer CD4-T-Zellen im Vergleich zur adjuvansbehandelten Kontrollgruppe [9]. Die P18-reaktiven CD4-T-Zellen waren überwiegend $\mathrm{T}_{\text {reg }}$ s und produzierten IL-10, wodurch atherosklerotische Läsionen in ApoE-defizienten Mäusen signifikant reduziert wurden. Daher stellen Impfstoffe, welche antigenspezifische $\mathrm{T}_{\text {regs }} \mathrm{s}$ induzieren, einen neuen und praktikablen Ansatz zur Verhinderung der Atherosklerose dar. Neben der Identifizierung von Antigenen sind geeignete Adjuvanzien und Administrationsrouten zur Entwicklung einer erfolgreichen Impfung notwendig. Dasselbe Apo- $\mathrm{B}_{100^{-}}$ Peptid kann bei geeignetem Adjuvans atheroprotektiv [14] und bei proinflammatorischer Kombination proatherosklerotisch sein [15]. Ein wichtiger Schritt in der Entwicklung einer human anwendbaren Impftherapie gegen Atherosklerose wurde mit der Immunisierung atherosklerotischer Mäuse mit Apo-B ${ }_{100^{-}}$ Peptiden, formuliert in $\operatorname{AddaVax}^{\mathrm{m}}$, getan. AddaVax ${ }^{\text {rs }}$ ist ein Adjuvans, welches auf einem Squalenöl basiert und dem klinisch zugelassenen MF59 ähnelt und erfolgreich atherosklerotische Läsionen verringert hat [12].

Atherosklerose ist eine chronisch inflammatorische Erkrankung und weist Autoimmunkomponenten auf. Gegen Komponenten des LDL-Cholesterols gerichtete Antikörper und CD4-T-Zellen sind bereits in gesunden Individuen vorhanden. Diese Immunreaktionen sind jedoch stark in der Progression der Atherosklerose, aber auch in die Atheroprotektion involviert. Die Induzierbarkeit von $\mathrm{T}_{\text {reg }}$ und die gleichzeitige Verhinderung der Atherosklerose in Tiermodellen legt nahe, dass die Impfung mit einem Apo-B-Peptid eine durchaus realistische Entwicklung zur Prävention der Atherosklerose darstellt. Dies erfordert jedoch Studien zur Dauer der Atheroprotektion nach erfolgter Impfung, zur Applikationshäufigkeit und zum Verabreichungsweg eines potenziellen Impfstoffs.

Es wird spannend sein $\mathrm{zu}$ sehen, ob in Zukunft in Addition zur etablierten und so erfolgreichen LDL-CholesterinSenkung auch immunologische Therapien zur Prävention von Herz-KreislaufErkrankungen ihre Anwendung finden.

\section{Korrespondenzadresse}

\section{H. Winkels}

La Jolla Institute for Immunology

La Jolla, CA, USA

holger@lji.org

Interessenkonflikt. H. Winkels und S. Baldus geben an, dass kein Interessenkonflikt besteht.

\section{Literatur}

1. Ridker PM, Danielson E, Fonseca FA et al (2008) Rosuvastatin to prevent vascular eventsinmen and women with elevated c-reactive protein. $\mathrm{N}$ Engl J Med 359:2195-2207

2. Ridker PM, Everett BM, Thuren Tet al (2017) Antiinflammatory therapy with canakinumab for atherosclerotic disease. N Engl J Med 377:1119-1131

3. Tabas I, Lichtman AH (2017) Monocyte-macrophages and t cells in atherosclerosis. Immunity 47:621-634

4. Binder CJ, Hartvigsen K, Chang MK et al (2004) II-5 links adaptive and natural immunity specific for epitopes of oxidized Idl and protects from atherosclerosis. JClin Invest 114:427-437

5. Que X, Hung MY, Yeang C et al (2018) Oxidized phospholipids are proinflammatory and proatherogenic in hypercholesterolaemic mice. Nature 558:301-306 
6. Paulsson G, Zhou X, Tornquist E, Hansson GK (2000) Oligoclonal t cell expansions in atherosclerotic lesions of apolipoprotein e-deficient mice. Arterioscler Thromb Vasc Biol 20:10-17

7. Koltsova EK, Garcia Z, Chodaczek G et al (2012) Dynamic $t$ cell-apc interactions sustain chronic inflammation in atherosclerosis. J Clin Invest 122:3114-3126

8. Hermansson A, Ketelhuth DF, Strodthoff D et al (2010) Inhibition of $t$ cell response to native lowdensity lipoprotein reduces atherosclerosis. J Exp Med 207:1081-1093

9. Kimura T, Kobiyama K, Winkels $\mathrm{H}$ et al (2018) Regulatory cd4(+) t cells recognize mhc-iirestricted peptide epitopes of apolipoprotein $b$. Circulation 138(11):1130-1143. https://doi.org/ 10.1161/circulationaha.117.031420

10. Gistera A, Hermansson A, Strodthoff D et al (2017) Vaccination against t-cell epitopes of native apob100 reduces vascular inflammation and disease in a humanized mouse model of atherosclerosis. J Intern Med 281:383-397

11. Lehrer-Graiwer J, Singh $P$, Abdelbaky $A$ et al (2015) Fdg-pet imaging for oxidized Idl in stable atherosclerotic disease: A phase ii study of safety, tolerability, and anti-inflammatory activity. Jacc Cardiovasc Imaging 8:493-494

12. Kobiyama K, Vassallo M, Mitzi J et al (2018) A clinically applicable adjuvant for an atherosclerosis vaccine in mice. Eur JImmunol 48:1580-1587

13. Kimura T, Tse K, McArdle $S$ et al (2017) Atheroprotective vaccination with mhc-ii-restricted apob peptides induces peritoneal il-10-producing cd4 t cells. Am J Physiol Heart Circ Physiol 312:H781-H790

14. Tse K, Gonen A, Sidney J et al (2013) Atheroprotective vaccination with mhc-ii restricted peptides from apob-100. Front Immunol 4:493. https://doi. org/10.3389/fimmu.2013.00493

15. Shaw MK, Tse KY, Zhao X et al (2017) T-cells specific for a self-peptide of apob-100 exacerbate aortic atheroma in murine atherosclerosis. Front Immunol 8:95. https://doi.org/10.3389/fimmu. 2017.00095

\section{Angriff von der Seite hemmt Teilchenfluss}

Zielgerichtete Blockade aus dem Hinterhalt: Ein neu entdeckter Mechanismus, mit dem sich lonenkanäle zielgerichtet blockieren lassen, weckt die Hoffnung auf Behandlungsmöglichkeiten bei Vorhofflimmern und weiteren Erkrankungen.

Zielgerichtete Blockade aus dem Hinterhalt: Ein neu entdeckter Mechanismus, mit dem sich lonenkanäle zielgerichtet blockieren lassen, weckt die Hoffnung auf Behandlungsmöglichkeiten bei Vorhofflimmern und weiteren Erkrankungen. Das schreibt eine Forschungsgruppe unter Marburger Leitung im Wissenschaftsmagazin "eLife“. Das Team ist auch an einem aktuellen Aufsatz in der Zeitschrift "Science" beteiligt, in dem es ebenfalls um die molekulare Steuerung von Kaliumkanälen geht.

Ob Herzrhythmusstörungen oder Atemstillstände während des Schlafs - so unterschiedlich diese Krankheitsbilder auch sind, eines haben sie gemeinsam: Sie lassen sich wahrscheinlich durch lonenkanäle beeinflussen, die für die elektrische Erregbarkeit der Zellen zuständig sind, speziell die sogenannten TASK-Kanäle. „Bislang gibt es keine Medikamente, die genau diese lonenkanäle zielgerichtet blockieren", erklärt der Physiologe Prof. Dr. Niels Decher von der PhilippsUniversität Marburg, der die Studie leitete. „Daher besteht ein hoher medizinischer Bedarf, neue Angriffspunkte an diesen Kanälen zu finden und neuartige Mechanismen zu entdecken, die zu ihrer Blockade führen." Viele unserer Körperfunktionen beruhen auf elektrischen Signalen, etwa die Nerventätigkeit oder der Herzschlag. Die elektrischen Signale kommen dadurch zustande, dass sich im Inneren von Zellen andere lonen befinden als außerhalb. Für die ungleiche Verteilung sorgen Kanäle, durch die lonen von einer Seite der Membran auf die andere gelangen; auf diese Weise baut sich ein elektrisches Potenzial über der Membran auf, die jede Zelle umhüllt.

Die Forschungsgruppe verglich verschiedene Wirkstoffe, die lonenkanäle vom TASK-Typ hemmen. Dabei zeigte das Narkosemittel Bupivakain eine unerwartete Wirkung, das in der Medizin zur örtlichen Betäubung eingesetzt wird: „Überraschenderweise blockiert Bupivakain vorzugsweise den nach außen gerichteten Teilchenstrom des Kanals, sobald sich das Membranpotenzial ändert", berichtet PD Dr. Susanne Rinné aus Dechers Arbeitsgruppe, eine der beiden Erstautoren des wissenschaftlichen Aufsatzes. Dergleichen wurde bisher noch nicht beobachtet. Das Team suchte anschließend nach der Stelle, an der Bupivakain ansetzt, wenn es den Kanal blockiert. Die Wissenschaftler nutzten hierfür molekulare Techniken, aber auch computergestützte Simulationsverfahren. Das Ergebnis: Das Narkosemittel greift an einer seitlichen Einbuchtung an, die von der zentralen Durchflussöffnung des Kanals abzweigt. „Erstaunlicherweise befindet sich das Medikament so weit seitlich in dieser Höhlung, dass es keinen Porenverschluss des Zentralkanals verursacht", erläutert Rinnés Kollege Dr. Aytuğ K. Kiper, der sich mit ihr die Erstautorenschaft teilt

"Was wir entdeckt haben, ist ein ganz neuer Mechanismus, der noch nie zuvor geschildert wurde", legt Decher dar. Bisherige KanalHemmstoffe greifen bevorzugt an der zentralen Tunnelöffnung an; da diese bei allen Kanaltypen vorkommt, wirken solche Substanzen nicht zielgerichtet, sondern auf alle Kanaltypen gleichermaßen. „Die von uns entdeckte Bindungsstelle und der bislang unbekannte Hemm-Mechanismus können künftig dazu verwendet werden, zielgerichtete Kanalblocker zu finden, die dringend für die Therapie des Atemstillstands im Schlaf und des Vorhofflimmerns benötigt werden", führt der Studienleiter aus.

Fast gleichzeitig berichtet ein internationales Forschungsteam in der Wissenschaftszeitschrift "Science“ über einen molekularen Mechanismus, der die Öffnung von Kaliumkanälen unterschiedlichen Typs bewirkt. Auch an dieser Veröffentlichung hat das Marburger Team mitgewirkt.

Originalveröffentlichungen: Rinné S et al. The molecular basis for an allosteric inhibition of $\mathrm{K+-}$-flux gating in K2P channels. eLife. 2019, https://doi.org/10.7554/eLife.39476 Schewe M et al. A Pharmacological Master Key Mechanism that Unlocks the Selectivity Filter Gate in K+ Channels. Science. 2019;363:875-880 